\title{
Doze anos de evolução da Iniciativa Hospital Amigo da Criança no Brasil
}

\author{
Maria de Fátima Moura de Araújo e Bethsáida de Abreu Soares Schmitz²
}

Como citar Araújo MFM, Schmitz BAS. Doze anos de evolução da Iniciativa Hospital Amigo da Criança no Brasil. Rev Panam Salud Publica. 2007;22(2):91-9.

RESUMO Objetivo. Analisar a evolução da Iniciativa Hospital Amigo da Criança no Brasil de 1992 a 2004.

Métodos. Este estudo descritivo retrospectivo investigou o número de Hospitais Amigos da Criança (HAC) credenciados por ano de 1992 a 2004, sua distribuição por estado e região, o número de municípios com HAC e o número de partos ocorridos em 294 HAC do Sistema Único de Saúde (SUS) em 2004. Os dados foram obtidos em consulta ao Sistema de Informações Hospitalares do Ministério da Saúde, às Secretarias Estaduais de Saúde e em relatórios da Área de Aleitamento Materno do Ministério da Saúde.

Resultados. De 1992 a 2004 foram credenciados 312 HAC no Brasil, distribuídos em 24 estados e no Distrito Federal. Desses, um foi descredenciado e 10 foram desativados. A distribuição regional dos 301 HAC existentes em 2004 foi: 139 no Nordeste, 59 no Sudeste, 50 no Sul, 37 no Centro-Oeste e 16 no Norte. Nos anos de 1997, 2003 e 2004 ocorreram quedas bruscas no credenciamento de HAC no País. Em 2004, dos 4347 hospitais com leitos obstétricos do SUS, 294 (6,8\%) eram HAC. Dos 3346 municipios onde existem hospitais com leitos obstétricos do SUS, 205 (6,1\%) possuem HAC. Ocorreram 2227971 partos nos hospitais do SUS em 2004, e desses, 565990 (25,4\%) foram realizados nos HAC.

Conclusão. O número de HAC é muito pequeno quando comparado ao número de hospitais com leitos obstétricos do SUS no Brasil. A desaceleração no credenciamento e a desativação de HAC registrados, sobretudo nos últimos anos, alertam para a necessidade de iniciativas para expandir e manter os HAC no País.

Palavras-chave Aleitamento materno, Brasil, política de saúde, Hospital Amigo da Criança.

A Iniciativa Hospital Amigo da Criança (IHAC), criada em 1990 pela Organização Mundial da Saúde (OMS)

\footnotetext{
Secretaria de Saúde do Distrito Federal, Fundação de Ensino e Pesquisa em Ciências da Saúde, Escola Superior de Ciências da Saúde, Coordenação de Pós-Graduação. Enviar correspondência para esta autora no seguinte endereço: SQN 215, Bloco J, apto. 205, CEP 70874-100, Brasília, DF, Brasil. Fone: +55-61-3349.7945. E-mail: mfmaraujo@terra.com.br

2 Universidade de Brasília (UnB), Faculdade de Ciências da Saúde, Departamento de Nutrição, e Observatório de Políticas de Segurança Alimentar e Nutrição/Núcleo de Pesquisa em Políticas Públicas.
}

e pelo Fundo das Nações Unidas para a Infância (UNICEF), tem como objetivo promover, proteger e apoiar o aleitamento materno. A base da iniciativa são os "Dez Passos para o Sucesso do Aleitamento Materno" $(1,2)$-usados para capacitar toda a equipe hospitalar que trabalha com mães e bebês para informar sobre as vantagens e o correto manejo do aleitamento materno e sobre as desvantagens do uso dos substitutos do leite materno, das mamadeiras e das chupetas, entre outros $(1,3)$.
No Brasil, o Ministério da Saúde, por meio do Programa Nacional de Incentivo ao Aleitamento Materno (PNIAM) e do Grupo de Defesa da Saúde da Criança, com o apoio do UNICEF e da Organização Pan-Americana da Saúde (OPAS), iniciou, em março de 1992, a implantação da IHAC. Em 1996, foram agregados ao processo de credenciamento de hospitais cinco requisitos (4-6), revisados em 2001 (7): não estar respondendo à sindicância do Sistema Único de Saúde (SUS); dispor de res- 
ponsável habilitado para a assistência à mulher e à criança na maternidade e na sala de parto; possuir comissão de estudo de morbi-mortalidade materna e neonatal implantada e atuante; apresentar taxa de cesariana conforme o estabelecido pela secretaria estadual de saúde, tendo como referência as regulamentações do Ministério da Saúde; e praticar a permanência hospitalar mínima de 24 horas para parto normal e de 48 horas para parto cesáreo (7).

Em 2004, os requisitos foram novamente modificados (8). Atualmente, são 10 os requisitos a serem atendidos para habilitação na IHAC. É importante notar que o Brasil é o único país no mundo a exigir o cumprimento desses requisitos no processo de credenciamento de Hospitais Amigos da Criança (HAC).

Dessa forma, o processo de credenciamento na IHAC, no Brasil, inicia-se com o preenchimento, pelo hospital, do questionário de auto-avaliação. Esse instrumento é encaminhado pela secretaria municipal de saúde à área competente da secretaria estadual de saúde, que, em seguida, realiza a préavaliação do hospital. Quando o hospital passa nessa etapa, a secretaria estadual solicita ao Ministério da Saúde a avaliação global da unidade, que é realizada por dois avaliadores credenciados pelo Ministério, sendo um deles de origem externa à região que está sendo avaliada. Os resultados da avaliação global são encaminhados à área competente do Ministério da Saúde para análise final $(7,8)$. Quando o hospital atende a todos os "Dez Passos" da IHAC e a todos os requisitos, é credenciado como $\operatorname{HAC}(5,8)$.

Para o sucesso do credenciamento, alguns fatores são fundamentais: o treinamento de toda a equipe que trabalha com mães e bebês (9) e a sensibilização do dirigente do hospital e das chefias de serviços da maternidade (3, 10). No Brasil, para acelerar o processo de credenciamento, foram realizados, de 2000 a 2002, 42 cursos para gestores de maternidades em 24 estados. Nesse período, foram treinados 1819 diretores e chefias de unidades de 859 hospitais e maternidades $(11,12)$. Os planos de ação desenvolvidos pelos partici- pantes dos cursos foram acompanhados nos anos de 2001 e 2002 por meio das reuniões de seguimento $(11,12)$, cujo objetivo foi apoiar as instituições e as secretarias estaduais de saúde na resolução dos problemas identificados, visando a aumentar o credenciamento de HAC no País.

Entretanto, não existem muitos estudos que avaliem o impacto da IHAC em nível populacional. Os resultados disponíveis mostram taxas de aleitamento materno mais expressivas na presença da estratégia (13). Uma pesquisa sobre o impacto e a eficiência do programa desenvolvido pelos HAC, realizada em Porto Alegre, no Brasil, mostrou uma duração mediana de aleitamento materno exclusivo (AME) de 1 e 2 meses, respectivamente, nas crianças nascidas antes e depois que o hospital se tornou "Amigo da Criança" (14). Um estudo populacional regional, também do Brasil, indicou que a duração mediana do aleitamento materno passou de 8,9 para 11,6 meses, e a do AME passou de 27 dias para 3,5 meses após a implantação da IHAC em um município (15).

Entre diversas intervenções que poderiam levar à redução da mortalidade infantil, o aleitamento materno exclusivo se destaca como a de melhor potencial para salvar vidas, associado ao aleitamento contínuo no primeiro ano de vida. Estima-se que, sozinha, essa intervenção poderia prevenir 1 em cada 7,5 mortes infantis (16). Além da sua ação direta sobre a sobrevivência infantil, destacam-se os efeitos benéficos da amamentação a longo prazo. Uma recente revisão da literatura indicou que os indivíduos amamentados têm menores níveis de pressão sangüínea e colesterol total, assim como melhores resultados em testes de inteligência. Também foi observada uma menor prevalência de sobrepeso/obesidade e diabetes tipo 2 em crianças que receberam aleitamento materno (17).

A IHAC, portanto, destaca-se como uma estratégia de grande relevância para mudar as rotinas e as práticas hospitalares referentes ao aleitamento materno, contribuindo de forma contundente para a melhoria dos indica- dores de aleitamento materno e, conseqüentemente, para a sobrevivência e garantia de melhores condições de saúde das crianças.

No entanto, em um país continental como o Brasil, é necessário conhecer não somente a distribuição de hospitais credenciados nessa Iniciativa, mas também a capacidade instalada de leitos obstétricos nos HAC, para se ter uma maior compreensão da potencial população a ser beneficiada por esse programa. A investigação e o conhecimento acerca do número de hospitais ainda candidatos a ingressar na IHAC no Brasil também são de extrema importância, no sentido de se buscarem estratégias e intervenções que possibilitem um maior avanço na velocidade de credenciamento de hospitais na Iniciativa.

O presente estudo teve como objetivo analisar a evolução do credenciamento de HAC desde a implantação da Iniciativa no Brasil, de forma a subsidiar processos de planejamento, gestão e avaliação de políticas de saúde voltadas para a atenção materno-infantil no País.

\section{MATERIAIS E MÉTODOS}

O presente estudo descritivo e retrospectivo analisa dados acerca da implementação da IHAC no Brasil entre 1992 a 2004 . Os dados foram coletados dos relatórios de 1999 a 2002 do Ministério da Saúde para avaliação das ações em aleitamento materno, do banco de dados do Sistema de Informações Hospitalares (SIH) do Ministério da Saúde, junto às secretarias estaduais de saúde e, em 2003 e 2004, da Área da Criança do Ministério da Saúde, que disponibilizou informações sobre os HAC. A pesquisa teve o apoio da equipe de suporte operacional dos sistemas da Secretaria de Atenção à Saúde do Ministério da Saúde para a coleta de dados sobre cada hospital e sobre a distribuição dos hospitais em cada município brasileiro, já que os dados disponíveis no $\mathrm{SIH}$ se referem ao total da rede hospitalar do SUS por estado e região brasileira, ou seja, não estão disponibilizados os dados desa- 
gregados. Dessa forma, a equipe do suporte operacional dos sistemas acessou as informações necessárias para este estudo diretamente no cadastro das instituições vinculadas.

As variáveis investigadas foram: número de HAC credenciados por ano nos estados no período de 1992 a 2004; número de hospitais com leitos obstétricos do SUS; distribuição de HAC vinculados ao SUS, por estado e região, em 2004; número de municípios que dispõem de hospitais com leitos obstétricos do SUS e que contam com HAC; e número de partos ocorridos nos HAC e nos hospitais com leitos obstétricos do SUS em 2004. Os hospitais com leitos obstétricos do SUS foram incluídos por serem potenciais candidatos à implantação da IHAC. Os dados de hospitais com leitos obstétricos do SUS e de municípios que contam com esses hospitais foram extraídos dos arquivos reduzidos de autorização de internação hospitalar (AIH), com o apoio da equipe técnica do SIH.

Para a análise comparativa da relação entre o número de HAC e o número de hospitais com leitos obstétricos do SUS no País, foram incluídos apenas os hospitais vinculados ao SUS em 2004 e que constavam na base de dados do SIH. Como o SIH não inclui dados dos hospitais militares e dos hospitais privados que não se vincularam ao SUS, não foi possível a realização do estudo em todo o universo da rede hospitalar no Brasil. Foram excluídos, portanto, desta análise, três HAC totalmente privados (dois no Estado do Maranhão e um no Estado de São Paulo) e quatro HAC das Forças Armadas (estados do Ceará, Paraíba, Rio Grande do Norte e Rio de Janeiro). Os resultados foram distribuídos por município, estado e região, expressos em números absolutos e porcentagem.

Para avaliar a evolução do número de HAC de 1992 a 2004, inicialmente foi realizada uma transformação logarítmica neperiana (18) na variável dependente número de HAC credenciados por ano. Com essa análise, foi possível verificar se o número de HAC cresceu ou decresceu, já que a variável apresentou, no período de 1992 a 2004, uma oscilação muito grande. A trans- formação revelou que os valores do número de HAC mostraram uma tendência de polinômio. Foram realizadas análises de tendência onde polinômios de primeiro, segundo e terceiro grau foram ajustados. Verificou-se que a função polinomial de segundo grau era aquela que melhor se ajustava aos dados. Foram obtidos um coeficiente linear igual a 0,784 $(P<0,001)$ e um coeficiente quadrático igual a $-0,049(P=$ 0,002). O ano (1992 a 2004) foi considerado como variável independente. Assim sendo, o modelo proposto foi: $\log \mathrm{n}^{\circ} \mathrm{HAC}=\beta_{0}+\beta_{1}$ ano $+\beta_{2}$ ano $^{2}$.

Para efeitos da análise estatística, foi considerado um nível de significância de $5 \%$.

\section{RESULTADOS}

Entre 1992 e 2004, foram credenciados no Brasil 312 HAC. Ao longo desse período, um HAC foi descredenciado e 10 foram desativados; portanto, 301 HAC estavam em funcionamento no Brasil em 2004 (tabela 1). A figura 1 mostra que em 1997, 2003 e 2004 ocorreram quedas bruscas no credenciamento, quando apenas 16,38 e 15 hospitais, respectivamente, foram credenciados. $\mathrm{O}$ ano de maior ingresso de HAC foi 2002, quando 57 hospitais foram certificados.

O modelo de regressão polinomial elaborado (figura 2) permitiu explicar $81,08 \%$ do comportamento da variável número de HAC credenciados por ano no período estudado. Nesse modelo, o número médio de HAC credenciados, dado pela exponencial $(0,78422)$, foi de 6,08 ao ano $(P<0,001)$. Observou-se ainda uma concavidade de $-0,049$, significativamente diferente de zero $(P=$ $0,002)$, o que indica uma tendência de desaceleração no período total, caracterizada por crescimento positivo nos anos de 1992 a 1996, desaceleração nos anos intermediários de 1997 a 2002 e decréscimo a partir de 2003.

$\mathrm{Na}$ análise regional, destacou-se o Nordeste, sobretudo em 1995 e 1996, quando foram credenciados 18 e 31 HAC, respectivamente $69,2 \%$ e $79,5 \%$ do total de HAC credenciados no Brasil nesse período. A Região Norte apresentou uma evolução mais lenta, sendo que Roraima e Rondônia até hoje não possuem HAC. Essa região apresentou, em 2004, a menor freqüência de HAC do País, de apenas 5,3\% (tabela 1).

Do total de 4347 hospitais com leitos obstétricos do SUS em 2004, 294 $(6,8 \%$ ) eram HAC (tabela 2$)$. As regiões Nordeste $(8,8 \%)$ e Centro-Oeste $(7,7 \%)$ apresentaram as maiores freqüências de HAC no total de hospitais com leitos obstétricos do SUS, enquanto o Norte apresentou a menor, com $4,4 \%$. Dos 3346 municípios brasileiros que possuíam hospitais com leitos obstétricos do SUS, $205(6,1 \%)$ contavam com HAC. As regiões Centro-Oeste (8,5\%) e Nordeste $(8,1 \%)$ apresentaram também as maiores proporções de municípios com HAC.

O Distrito Federal, onde se localiza a capital do País, destacou-se com 85\% dos seus hospitais com leitos obstétricos do SUS credenciados na IHAC (tabela 2). Os estados que apresentaram as maiores freqüências de municípios com HAC e de HAC na rede hospitalar do SUS foram: Alagoas, Amapá, Ceará, Paraíba, Rio Grande do Norte e Santa Catarina.

Foram realizados 2227971 partos hospitalares nos 4347 hospitais do SUS. Desse total, 25,4\% aconteceram nos 294 HAC com leitos obstétricos do SUS. Na análise regional destacou-se o Centro-Oeste, com 38,6\% de partos ocorridos nos HAC, sendo que o Distrito Federal teve considerável participação nesse resultado, com aproximadamente $94 \%$ do total dos partos do SUS realizados nos seus 11 HAC (tabela 2).

\section{DISCUSSÃO}

Neste estudo, a base de dados do SIH não representa o universo da rede hospitalar no Brasil. O SIH não permite a obtenção dos dados referentes aos hospitais militares e àqueles totalmente privados, sem vínculo com o SUS, não existindo, até o momento, nenhum outro tipo de rede de informação atualizada para captação desses dados. Contudo, acreditamos que os 
TABELA 1. Número de Hospitais Amigos da Criança credenciados por ano de 1992 a 2004 e total de Hospitais Amigos da Criança nos estados e regióes brasileiras em 2004

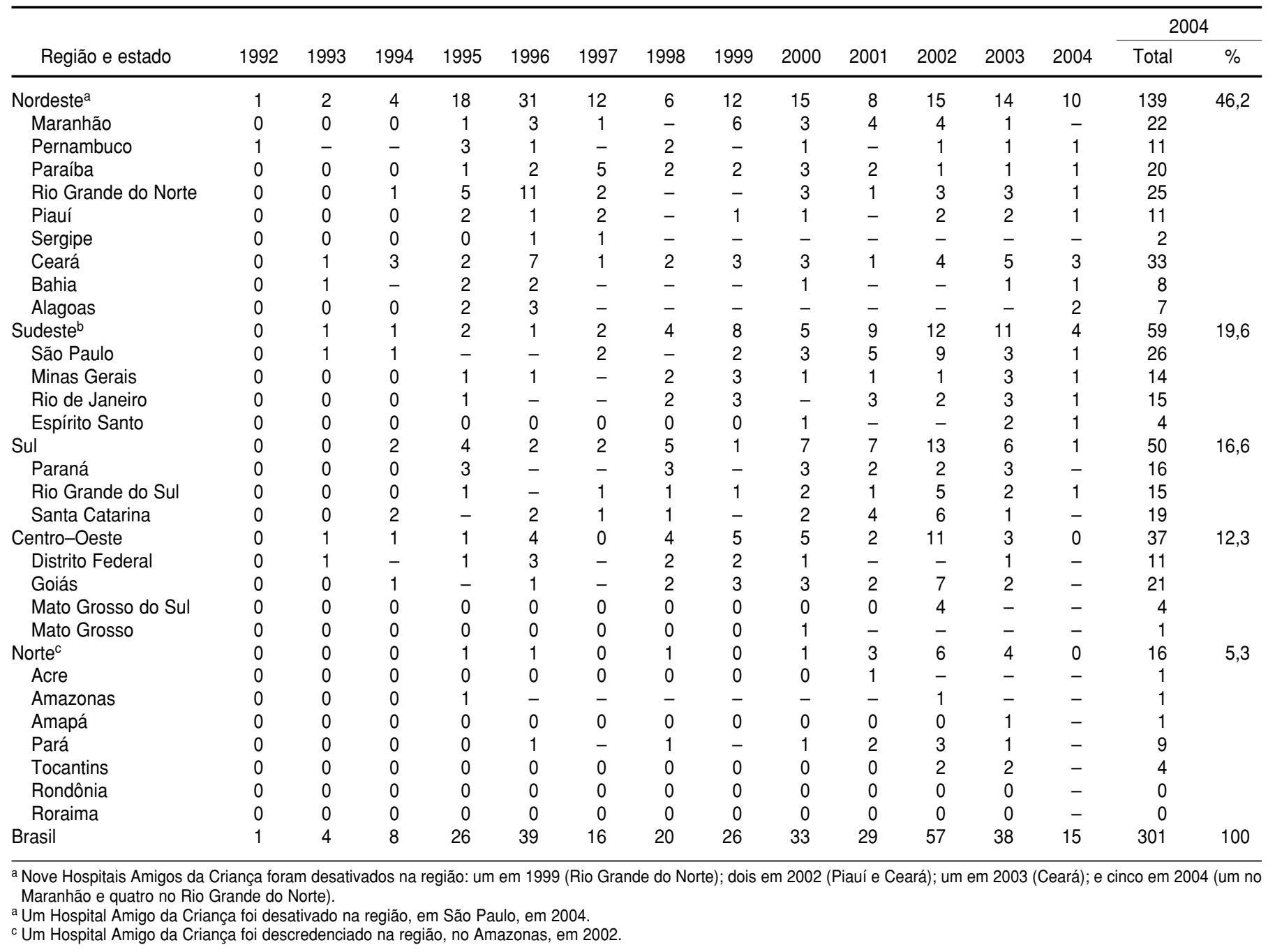

dados levantados sejam representativos: o SUS possui a maior rede hospitalar de atenção a mães e filhos no País, contando com mais de 4000 maternidades. Outra limitação é a inexistência de dados desagregados dos estabelecimentos cadastrados on-line no SIH, uma dificuldade que foi, contudo, superada com o apoio da equipe do Suporte Operacional dos Sistemas do Ministério da Saúde.

Este estudo mostrou um pequeno número de HAC no Brasil em comparação ao total de 4347 hospitais com leitos obstétricos do SUS existentes no País em 2004, além de uma tendência de desaceleração no credenciamento dos HAC, de forma mais crítica a partir de 1997. Como não há na literatura estudo similar, que tenha avaliado o perfil do credenciamento de HAC ao longo dos anos, não é possível realizar comparações com outras pesquisas. Sabe-se apenas que a IHAC tem avançado, com lentidão, na maioria dos países (10).

Em 12 anos da IHAC foram credenciados apenas 312 HAC no Brasil. Ao longo desse período, aconteceram quedas bruscas no credenciamento de hospitais, a primeira em 1997, de 59\% em comparação ao número de hospitais credenciados no ano anterior. Naquele ano, dois fatos parecem ter in- fluenciado negativamente os novos credenciamentos: a inclusão dos cinco requisitos, sendo um deles o que estabelece o limite máximo para a taxa de cesariana, de $30 \%$ para hospitais gerais e de $40 \%$ para hospitais de referência na assistência materno-infantil $(4,6)$, e a extinção do Instituto Nacional de Alimentação e Nutrição (INAN), onde o PNIAM estava inserido, tendo sido posteriormente integrado à Área de Saúde da Criança do Ministério da Saúde (12).

Na tabela 1 observa-se que em 1995 e 1996, quando os hospitais eram avaliados apenas quanto ao cumprimento dos "Dez Passos", como é preconizado 
FIGURA 1. Número de Hospitais Amigos da Criança credenciados por ano no Brasil, 1992 a 2004

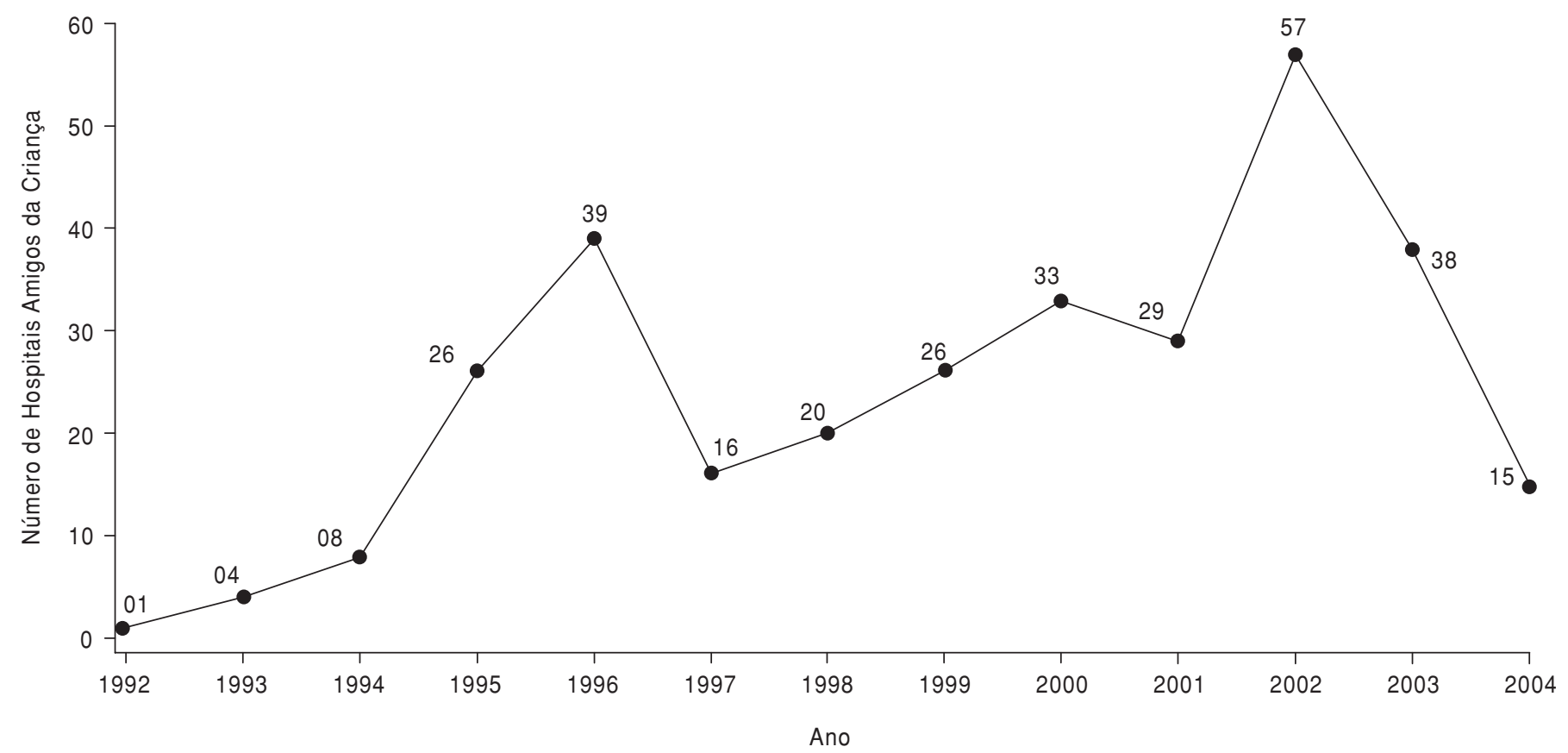

FIGURA 2. Curva ajustada da evolução do logaritmo do número de Hospitais Amigos da Criança credenciados por ano no Brasil, 1992 a 2004

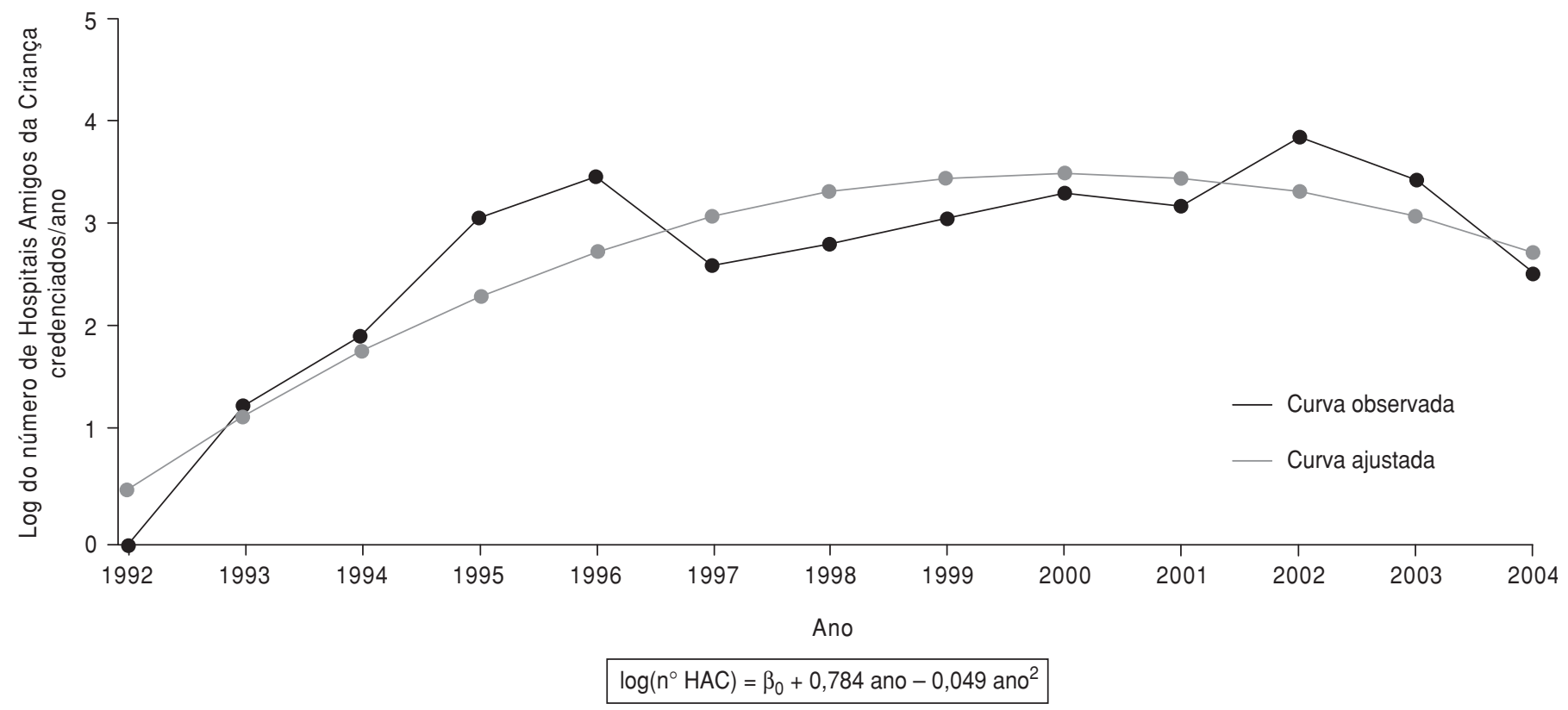

internacionalmente pelo UNICEF e a OMS, foram titulados 18 e 31 HAC no Nordeste. Em 1997, após a inclusão dos cinco requisitos, houve uma queda de $61,3 \%$ no credenciamento de hospi- tais nessa região em relação ao ano anterior.

Em 1998, ocorreu um incremento de $25 \%$ no número de hospitais credenciados, fruto da intensificação do trei- namento de equipes hospitalares, sendo essa tendência mantida até 2001, quando houve nova redução de $12,1 \%$ na certificação de hospitais, provavelmente em função dos limites 
TABELA 2. Distribuição dos leitos obstétricos e partos no SUS e em Hospitais Amigos da Criança no Brasil, 2004

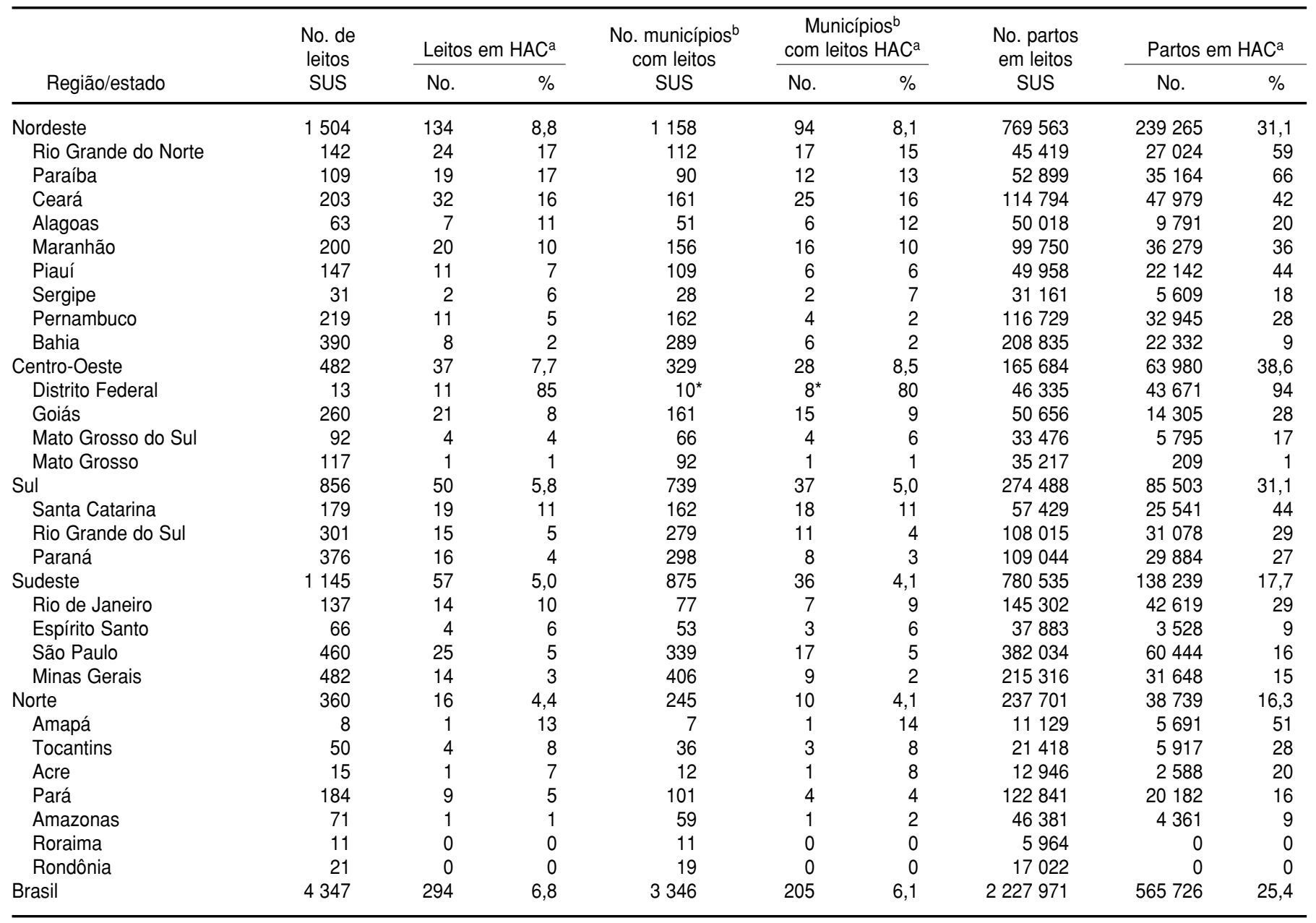

Fonte: Número de hospitais com leitos obstétricos do SUS, número de municípios com hospitais com leitos obstétricos do SUS e número de partos por ano: Suporte Operacional dos Sistemas da Secretaria de Atenção a Saúde do Ministério da Saúde.

${ }^{a} \mathrm{HAC}=$ Hospital Amigo da Criança. Porcentagens em relação aos dados do SUS.

${ }^{\text {b }} \mathrm{Ou}$, no caso do Distrito Federal, regiões administrativas.

mais baixos estabelecidos naquele ano para as taxas de cesariana. Por outro lado, na Austrália, um estudo concluiu que a cesariana foi uma barreira importante para a implementação do passo 4 da IHAC, ao revelar que mulheres com parto cesáreo apresentaram um retardo significativo na iniciação da amamentação quando comparadas com mulheres com parto vaginal com ou sem assistência instrumental (19). Todas essas informações devem ser pesadas. $\mathrm{O}$ passo 4 do processo de implantação da IHAC é considerado cumprido quando ocorre o contato pele a pele na primeira meia hora e a amamentação na primeira hora após o parto. Quando a equipe está bem treinada e sensibilizada, esse passo pode ser cumprido mesmo nos partos cesáreos. Portanto, deve-se, sim, limitar a cesárea, mas provavelmente não como um dos requisitos para credenciamento dos HAC; mesmo em hospitais onde o índice de cesariana é maior do que o estabelecido pelo Ministério da Saúde, se a equipe estiver orientada, é provável que as crianças serão beneficiadas com a amamentação.

Já as quedas registradas no credenciamento de hospitais em 2003 e em 2004 , de 33,3 e de $60,5 \%$, respectiva- mente, não podem ser explicadas pelo novo limite na taxa de cesariana ou por mudanças na estrutura organizacional do Ministério da Saúde, à semelhança do ocorrido em 1997 com a extinção do INAN. Verificou-se que, nesses anos, foram suspensos os cursos da IHAC para gestores de maternidades, assim como o seguimento desses cursos. A queda constatada em 2004 foi a maior registrada em todos os anos da IHAC no Brasil. Há evidências de que os cursos realizados em prol da IHAC são eficazes em mudar não só as práticas hospitalares, mas também o conhecimento dos profissionais de 
saúde, contribuindo para a melhoria das taxas de aleitamento materno (13, 20). Isso é corroborado pelo fato de que, entre os pilares de sustentação dessa estratégia, encontra-se a necessidade de motivação, formação e treinamento dos gestores e equipes de profissionais de saúde envolvidos nesse processo (15).

$\mathrm{O}$ ajuste dos dados de credenciamento de HAC confirmou a tendência de desaceleração e indicou um número médio de apenas seis hospitais credenciados por ano no período estudado. Esse dado, frente ao potencial de hospitais com leitos obstétricos do SUS, revela um baixo desempenho da política voltada para a implementação de HAC. Urge, portanto, promover o estabelecimento de intervenções eficientes, que permitam a expansão da IHAC para incluir todos os 4098 hospitais com leitos obstétricos do SUS existentes em 2004, bem como os hospitais totalmente privados ainda não credenciados na Iniciativa.

Uma análise mais detalhada do processo de credenciamento dos HAC indica que o Brasil, por ser o único a exigir o cumprimento de 10 requisitos além dos "Dez Passos", estabelece um processo administrativo mais complexo e de maior exigência operacional por parte das instituições hospitalares interessadas em aderir a essa estratégia. Esse fato, associado às dificuldades de manutenção e funcionamento dos hospitais públicos, e à suspensão da capacitação de gestores de hospitais, tem contribuído para uma baixa motivação e realização de novos credenciamentos (21).

Como a série analisada compreende um curto período de tempo, não se pode afirmar que o efeito decrescente observado deva ocorrer de forma semelhante nos anos posteriores a 2004, sendo necessário cuidado quanto a extrapolações futuras. Entretanto, dados mais recentes obtidos do Ministério da Saúde, referentes aos anos de 2005 (22) e 2006, indicam que apenas 18 e oito HAC foram credenciados, respectivamente, confirmando o efeito decrescente observado neste estudo.

$\mathrm{O}$ ano de 2002 destacou-se como o que mais certificou hospitais na Inicia- tiva (57 hospitais), com um incremento de $96,6 \%$ em comparação a 2001. Esse incremento parece ter sido resultado das estratégias estabelecidas e recomendadas pela OMS, a saber, os cursos para gestores de maternidades e as reuniões de seguimento $(11,12)$. Dos 97 hospitais certificados de 2000 a 2002, 79 (68\%), tiveram seus representantes capacitados nesse curso $(11,12)$. Entretanto, desde 2003 o Ministério deixou de promover a capacitação de gestores e de profissionais de saúde para a IHAC nos estados e municípios brasileiros, prejudicando consideravelmente a expansão do credenciamento de $\operatorname{HAC}(12,21)$. Em alguns hospitais, as mudanças de rotina podem ser lentas e difíceis. Apenas dirigentes e administradores de saúde bem informados e apoiados pelas secretarias municipais e estaduais de saúde, assim como pelo próprio Ministério da Saúde, conseguem promover as transformações necessárias para a implantação da IHAC.

Em 2004, dos 312 HAC certificados, apenas 301 (96,5\%) continuavam efetivamente na Iniciativa. Entre 1999 e 2004, 12 HAC fecharam todos os seus leitos obstétricos. Dois desses hospitais reativaram os seus leitos obstétricos posteriormente, ambos sem passar por novo processo, seja de avaliação global, seja de reavaliação dos "Dez Passos". Portanto, havia 10 HAC desativados em 2004. Em todos esses 10 casos, as secretarias municipais e estaduais de saúde não informaram o Ministério sobre a desativação, o que tornou o banco de dados do Ministério sobre a IHAC desatualizado no período. A desativação de três desses HAC foi constatada apenas durante o processo de análise dos formulários de reavaliação de HAC (21), enquanto que a desativação dos outros sete HAC foi detectada pela ausência no SIH de dados sobre partos hospitalares, tendo sido confirmada após consulta às respectivas secretarias estaduais de saúde. É imprescindível o estabelecimento de um sistema integrado de informações entre as áreas técnicas responsáveis pelos HAC nas secretarias estaduais de saúde e no Ministério da Saúde para fortalecer a confiabilidade das informações, bem como o incremento de ações conjuntas, no sentido de promover e apoiar a implantação e a manutenção de hospitais na Iniciativa. Apenas uma desativação de HAC foi detectada, pelo próprio Ministério, em 1999, também durante o processo de reavaliação de HAC.

Além dos 10 hospitais desativados, um HAC foi descredenciado durante o processo de reavaliação de hospitais, em 2002, por não cumprir nenhum dos "Dez Passos" (21). Isso parece ter ocorrido porque o hospital não contava com equipe médica própria. A equipe era oriunda de uma cooperativa médica, sem vínculo empregatício com o hospital, e a alta rotatividade dos médicos que atendiam a mãe e o bebê na maternidade impossibilitou o treinamento desses profissionais.

Excluindo os três HAC privados sem convênio com o SUS e os quatro HAC militares, não incluídos neste estudo, eram 294 os HAC do SUS em funcionamento no Brasil em 2004, o que equivale a apenas $6,8 \%$ do total de hospitais com leitos obstétricos do SUS. Uma comparação com dados internacionais permite verificar que, em 14 países europeus, a porcentagem de HAC em relação ao total de hospitais existentes foi superior a 10\% (23). Além disso, em 2001, a China já havia certificado 6312 HAC, a Nigéria 1147 e o México, 692 (23).

Analisando a distribuição dos HAC nas regiões brasileiras, o Distrito Federal destaca-se, apresentando, em 2004, $85 \%$ dos seus hospitais com leitos hospitalares do SUS credenciados na Iniciativa. Mato Grosso, Roraima e Rondônia apresentaram percentuais iguais ou inferiores a $1 \%$ de HAC e de municípios com HAC na rede hospitalar do SUS. Tais freqüências são insuficientes para fornecer atenção humanizada e especializada para a prática da amamentação. Além disso, os dados relativos à Região Norte, onde apenas $5,4 \%$ dos hospitais com leitos obstétricos do SUS são HAC, indicam que a dificuldade de acesso parece interferir no credenciamento, dificultando os treinamentos de equipes e de gestores.

Um estudo realizado por Venâncio et al. (24) concluiu que a ausência de 
HAC é um fator de risco para o desmame. $\mathrm{O}$ estudo constatou ainda que nascer em um município onde existe pelo menos um HAC mostrou associação significativa com os índices de aleitamento materno. É necessário, portanto, expandir a Iniciativa para mais municípios brasileiros, especialmente das regiões Norte, Sudeste e Sul.

Apesar de poucos, os 294 HAC são responsáveis por $25,4 \%$ dos nascimentos realizados nos hospitais com leitos obstétricos do SUS no Brasil. Como não tivemos acesso ao número de partos realizados nos hospitais não conveniados ao SUS, não foi possível comparar este estudo com o de Cattaneo, que observou que, em 19 países da Europa, menos de $15 \%$ dos nascimentos ocorriam em HAC (25).

São raros os HAC sem vínculo com o SUS no Brasil (2,3\%). Até 2004, apenas sete hospitais (três privados e quatro militares) haviam ingressado na Iniciativa. Toma e Monteiro observaram que, de modo geral, rotinas de proteção, promoção e apoio à amamentação foram encontradas com mais freqüência nos hospitais públicos do que nos privados de São Paulo (10). É preocupante que a grande maioria dos hospitais privados no Brasil não esteja incluída na IHAC. Mais estudos são necessários para se conhecer a freqüência real de partos ocorridos em HAC no Brasil, incluindo os dados de hospitais privados.
A grande maioria dos hospitais do SUS ainda não pratica os "Dez Passos" da Iniciativa, demonstrando que, apesar do consenso sobre a importância da amamentação natural, nem sempre as rotinas hospitalares vigentes têm contribuído para essa prática. Rotinas e práticas hospitalares inadequadas podem induzir ao desmame precoce, com repercussões no aumento da desnutrição, da diarréia, das doenças infecciosas, e assim, da morbi-mortalidade infantil $(2,26)$. Alguns estudos têm mostrado o impacto positivo dos princípios da Iniciativa no aumento da freqüência e da duração da amamentação $(14,27)$. A redução nas ações de promoção, proteção e apoio à amamentação pode levar ao decréscimo nos indicadores de aleitamento materno, e conseqüentemente, a um maior gasto com substitutos do leite materno e com a assistência à saúde $(3,28)$.

Uma ação prioritária para estimular a promoção e o apoio ao aleitamento materno no pré-natal e nas primeiras semanas e meses pós-parto é a implantação da Iniciativa Unidade Básica Amiga da Amamentação (IUBAAM) em âmbito nacional. Um exemplo bem sucedido dessa estratégia é a experiência do Estado do Rio de Janeiro (12, 26). A IUBAAM, elaborada nos anos de 2000 a 2002 por consultores do Ministério da Saúde (12), pode contribuir para o cumprimento dos passos 3 e 10 da IHAC, bem como para o credenciamento de novos HAC $(12,21)$.

O presente estudo representou uma primeira análise sobre o processo evolutivo da Iniciativa Hospital Amigo da Criança no Brasil, tendo concluído que o número de HAC ainda é inexpressivo quando comparado ao total de hospitais com leitos obstétricos existentes no País. O estudo indica que é urgente obter apoio do governo e dos gestores de saúde para implantar estratégias e ações complementares que promovam a expansão, a manutenção e a melhoria da qualidade da Iniciativa no Brasil. Os resultados também sugerem que é preciso discutir a viabilidade dos requisitos de credenciamento de HAC no Brasil, bem como reiniciar os cursos que orientam a Iniciativa, como recomenda a OMS $(2,3)$, para que a Iniciativa possa ter possibilidades concretas de continuidade.

Agradecimentos. Este estudo só pôde ser realizado com a colaboração da equipe do Suporte Operacional dos Sistemas da Secretaria de Atenção a Saúde do Ministério da Saúde. Agradecemos especialmente ao Professor Dr. Eduardo Freitas da Silva, do Departamento de Estatística da Universidade de Brasília, pela colaboração na definição e aplicação da análise estatística utilizada neste estudo.

\section{REFERÊNCIAS}

1. World Health Organization, United Nations Children's Fund. Innocent Declaration. Florença: UNICEF/WHO; 1990.

2. Organização Mundial da Saúde. Evidências científicas dos Dez Passos para o Sucesso do Aleitamento Materno. Brasília: Organização Pan-Americana da Saúde; 2001.

3. World Health Organization, Wellstart International. Promoting breast-feeding in health facilities. A short course for administrators and policy-makers. Genebra: WHO/Wellstart; 1996. (Document WHO/NUT 96.3).

4. Araújo MFM, Otto AFN, Schmitz BAS. Primeira avaliação do cumprimento dos "Dez Passos para o sucesso do aleitamento materno" nos hospitais Amigos da Criança do Brasil. Rev Bras Saúde Matern Infant. 2003;3(4):411-9.
5. Lamounier JA. Experiência Iniciativa Hospital Amigo da Criança. Rev Assoc Med Bras. 1998; 44(4):319-24.

6. Lamounier JA, Maranhão AGK, Araújo MFM. A Iniciativa Hospital Amigo da Criança no Brasil. Em: Rego JD. Aleitamento materno. São Paulo: Atheneu; 2001. Pp. 333-42.

7. Brasil, Ministério da Saúde. Portaria SAS $n^{\circ}$ 29 de 22 de junho de 2001. Estabelece as normas para o processo de credenciamento e de descredenciamento dos Hospitais Amigos da Criança no País. Diário Oficial da União 2001. 27 de junho:seção 1 (123-E):55.

8. Brasil, Ministério da Saúde. Portaria SAS $n^{\circ}$ 756 de 16 de dezembro de 2004. Estabelece as normas para o processo de habilitação do Hospital Amigo da Criança integrante do Sis- tema Único de Saúde (SUS). Diário Oficial da União 2004. 17 de dezembro:Seção 1 (242):99.

9. United Nations Children's Fund. Manejo e promoção do aleitamento materno: curso de 18 horas para equipes de maternidades. Brasília: UNICEF; 1993.

10. Toma TS, Monteiro CA. Avaliação da promoção do aleitamento materno nas maternidades públicas e privadas do município de São Paulo. Rev Saude Publica. 2001;35(5):409-14.

11. Brasil, Ministério da Saúde. Avaliação das ações executadas em aleitamento materno no ano de 2002: relatório. Brasília: Ministério da Saúde; 2002.

12. Araújo MFM. Situação e perspectivas do aleitamento materno no Brasil. Em: Carvalho MR, Tamez RN. Amamentação: bases científicas. 
Rio de Janeiro: Guanabara Koogan; 2005. Pp. 269-81.

13. Labbok MH. Aleitamento materno e a Iniciativa Hospital Amigo da Criança: mais importante e com mais evidências do que nunca. J Pediatr (Rio J). 2007;83(2):99-101.

14. Braun ML, Giugliani ER, Soares ME, Giugliani C, de Oliveira AP, Danelon CM. Evaluation of the impact of the baby-friendly hospital initiative on rates of breastfeeding. Am J Public Health. 2003;93(8):1277-9.

15. Caldeira AP, Gonçalves E. Assessment of the impact of implementing the Baby-Friendly Hospital Initiative. J Pediatr (Rio J). 2007; 83(2):127-32.

16. Jones G, Steketee RW, Black RE, Bhutta ZA, Morris SS; Bellagio Child Survival Study Group. How many child deaths can we prevent this year? Lancet. 2003;362(9377):65-71.

17. Horta B, Bahl R, Martines J, Victora C. Evidence on the long-term effects of breastfeedingsystematic reviews and meta-analyses. Genebra: WHO; 2007. Disponível em: www. who.int/child-adolescent-health / New Publication / NUTRITION/ISBN_92_4 159523_0.pdf. Acessado em julho de 2007.

18. Neter J, Wasserman W, Kutner MH. Polynomial regression. Em: Netyer J, Wasserman W,
Kutner MH. Applied linear regression models. Illinois: Irwin; 1983. Pp. 300-13.

19. Rowe-Murray HJ, Fisher JR. Baby friendly hospital practices: cesarean section is a persistent barrier to early initiation of breastfeeding. Birth. 2002;29(2):124-31.

20. Valdés V, Pugin E, Labbok MH, Pérez A, Catalan S, Aravena R, et al. The effects on professional practices of a three-day course on breastfeeding. J Human Lact. 1995;11(3):185-90.

21. Araújo MFM. Iniciativa Hospital Amigo da Criança no Brasil: análise da evolução do credenciamento de hospitais no período de 1992 a 2004 e da reavaliação do cumprimento dos "Dez passos para o sucesso do aleitamento materno", em 2002 [dissertação]. Brasília (DF); Universidade de Brasília; 2005.

22. Brasil, Ministério da Saúde. Saúde da Criança. IHAC. MS. Disponível em: http://www. portal.saude.gov.br/portal/saude/cidadao/ visualizar_texto.cfm?idtxt=24215. Acessado em 5 de maio de 2007.

23. Naylor AJ. Baby-Friendly Hospital Initiative. Protecting, promoting, and supporting breastfeeding in the twenty-first century. Pediatr Clin North Am. 2001;48(2):475-83.

24. Venâncio SI, Escuder MML, Kitoko P, Rea MF, Monteiro CA. Freqüência e determinan- tes do aleitamento materno em municípios do estado de São Paulo. Rev Saude Publica. 2002; 36(3):313-8.

25. Cattaneo A, Yngve A, Koletzko B, Guzman LR; Promotion of Breastfeeding in Europe project. Protection, promotion and support of breast-feeding in Europe: current situation. Public Health Nutr. 2005;8(1):39-46.

26. Oliveira MIC, Gomes MASM. As unidades básicas amigas da amamentação: uma nova tática no apoio ao aleitamento materno. Em: Rego JD. Aleitamento Materno. Rio de Janeiro: Atheneu; 2001. Pp. 343-66.

27. Kramer MS, Chalmers B, Hodnett ED Sevkovskaya Z, Dzikovich I, Shapiro S, et al. Promotion of breastfeeding intervention trial PROBIT): a randomized trial in the Republic of Belarus. JAMA. 2001;285(4):413-20.

28. Araújo MFM, Del Fiaco A, Pimentel LS, Schmitz BAS. Custo e economia da prática do aleitamento materno para a família. Rev Bras Saude Mater Infant. 2004;4(2):135-41.

Manuscrito recebido em 24 de julho de 2006. Aceito em versão revisada em 29 de maio de 2007.

ABSTRACT Objective. To evaluate implementation of the Baby-Friendly Hospital Initiative (BFHI) in Brazil from 1992 to 2004.

Methods. This retrospective descriptive study of the BFHI in Brazil examined the Twelve years of the Baby-Friendly Hospital Initiative in Brazil number of Baby-Friendly Hospitals (BFH) accredited per year from 1992 to 2004, state and regional distribution of the hospitals, the number of municipalities with a $\mathrm{BFH}$, and the number of births at the 294 BFH that were participating in Brazil's universal, public health care system (Serviço Único de Saúde) in 2004. Data were obtained from the Ministry of Health's Hospital Information System, from state health departments, and from Ministry of Health's reports on breastfeeding.

Results. From 1992 to 2004, a total of 312 hospitals were BFH-accredited across 24 of Brazil's 26 states and the Federal District. Of these, one had lost accreditation and 10 had been deactivated by the end of the study period. The regional distribution of the 301 remaining BFH in 2004 was: 139 in the Northeast, 59 in the Southeast, 50 in the South, 37 in the Midwest, and 16 in the North. A sharp drop in the accreditation rate was recorded in certain years: 1997, 2003, and 2004. In 2004, 294 (6.8\%) of the 4347 public hospitals with maternity beds were BFH. Of the 3346 municipalities that had public hospitals with maternity beds, 205 (6.1\%) also had BFH. In 2004, there were 2227971 births in public hospitals, of which 565990 (25.4\%) occurred in BFH.

Conclusion. The number of BFH in Brazil is relatively small when compared to the number of public hospitals with maternity beds. The decreased accreditation rate and the deactivation of accredited $\mathrm{BFH}$, especially in the latter years of the study, indicate the need for measures that will bolster and grow the BFHI in Brazil.

Key words Maternity hospital, breast feeding, health policy, nutrition policy, Brazil. 